\title{
Something is changing in adherence to CPAP therapy: real world data after 1 year of treatment in patients with obstructive sleep apnoea
}

\author{
To the Editor:
}

Obstructive sleep apnoea syndrome (OSA) is a common sleep disorder, affecting at least $24 \%$ of the male and $9 \%$ of the female population [1]. Despite progress in alternative treatment options, continuous positive airway pressure (CPAP) therapy is still the first-line treatment in moderate to severe OSA. The effectiveness of CPAP treatment is proportional to the usage: the greater the number of hours of CPAP use per night the greater the improvement in OSA symptoms, daytime sleepiness, quality of life, sleep architecture and blood pressure. Adherence to treatment, however, is the crucial issue: in many case series more than $50 \%$ of patients with OSA had interrupted treatment 1 year after the prescription [2].

Several studies have proposed different protocols or approaches to increase adherence, i.e. instructions to patients to manage the CPAP machine, humidifier and the mask, and psychological programmes, such as intensive sleep education, cognitive behavioural intervention, social cognitive therapy, motivational enhancement therapy, telemedicine intervention and behavioural treatment [3-8]. In spite of the major burden imposed it is still unclear if these interventions really increase the adherence to CPAP therapy in the real world and in the long term, although they usually obtain an improvement (but which is often marginal) in the number of hours of CPAP use (table 1). Cistulli et al. [9] recently studied a large cloud-based database of patients showing that the rate of adherence to CPAP therapy, defined as $4 \mathrm{~h}$ CPAP use on $70 \%$ of nights in a consecutive 30 -day period in the first 90 days of therapy, was $75 \%$, independent of machine type, suggesting that three quarters of patients in common practice are adherent to treatment.

In the light of these considerations, we decided to analyse CPAP adherence in consecutive OSAS patients in a clinical setting, according to our clinical practice. This is a retrospective monocentric observational study.

In our clinical setting, the first step is a home cardio-respiratory study; the results are communicated in a 30-min visit with a sleep certified physician where the patient is informed of OSA diagnosis and possible treatment options. Candidates for CPAP treatment undergo a 40-min session with a skilled technician who chooses a properly fitting mask and trains the patient in CPAP use, ramp adjustment and management of the humidifier. After 1 week of auto-CPAP training at home, a fixed CPAP prescription follows according to data obtained from the device and confirmed by a sleep study. The patient obtains a formal prescription of CPAP after attending a group educational session with a sleep expert technician lasting about $1 \mathrm{~h}$, characterised by introduction to respiratory sleep disorders, choice of treatment, CPAP machine management, and information on reimbursement policies and possible restrictions to driving licences. After 1 year of treatment a follow-up visit is scheduled, which includes an interview and assessment of CPAP usage from the built in data stored on the device. In case of need, the patient has the opportunity to contact the sleep laboratory before the scheduled visit.

We analysed data from consecutive OSA patients accessing our sleep laboratory between 2015 and 2017. During this period 1611 patients had a diagnosis of OSA, but 272 patients opted to undergo an alternative treatment. On the basis of the data downloaded from the device used at home, we divided the 1339 (338 female)

@ERSpublications

Adherence to CPAP therapy is effective when applying a standard protocol consisting of a diagnostic procedure, accurate pressure setting and mask choice, a group educational session on sleep disorders and 1-yr follow up visit http://bit.ly/352XPBH

Cite this article as: Morrone E, Giordano A, Carli S, et al. Something is changing in adherence to CPAP therapy: real world data after 1 year of treatment in patients with obstructive sleep apnoea. Eur Respir $J$ 2020; 55: 1901419 [https://doi.org/10.1183/13993003.01419-2019]. 
TABLE 1 Results of some protocols designed to enhance obstructive sleep apnoea patients' adherence to continuous positive airway pressure therapy

\begin{tabular}{|c|c|c|c|c|}
\hline Study & Subjects $n$ & Intervention & Adherence $\mathrm{h}$ per night & Time of follow-up \\
\hline BAKKER et al. [6], 2016 & 83 & $\begin{array}{l}\text { Motivational enhancement, two } 1-\mathrm{h} \text { in } \\
\text { person sessions plus six telephone calls } \\
\text { lasting } 10-30 \mathrm{~min}\end{array}$ & $4.4 \pm 2.9$ versus $3.3 \pm 2.7$ & 6 months \\
\hline PÉpIN et al. [8], 2018 & 306 & $\begin{array}{l}\text { Remote activity, sleep, BP oximetry and } \\
\text { adherence monitoring with coaching }\end{array}$ & $5.28 \pm 2.23$ versus $4.75 \pm 2.50$ & 6 months \\
\hline
\end{tabular}

Adherence data are expressed as mean \pm SD, intervention versus control group, unless otherwise stated. MET: motivational enhancement treatment; BP: blood pressure.

patients who accepted CPAP treatment into three groups: group 1 , poor adherence ( $\leqslant 3.9 \mathrm{~h}$ per night); group 2, acceptable adherence ( 4 to $4.9 \mathrm{~h}$ per night) and group 3, good adherence (at least $5 \mathrm{~h}$ per night; median $6.8 \mathrm{~h})$. No difference among these three groups was found regarding age $(60.6 \pm 11.4$ versus $62.5 \pm 10.9$ versus $61.4 \pm 11.3$ years, respectively), weight $(89.0 \pm 19.1$ versus $87.9 \pm 18.8$ versus $89.7 \pm 20.4 \mathrm{~kg})$, height $(169.4 \pm 8.4$ versus $169.1 \pm 8$ versus $168.7 \pm 8.9 \mathrm{~cm})$, apnoea/hypopnoea per hour $(34.0 \pm 20.8$ versus $37.1 \pm 21.4$ versus $40.7 \pm 24.2$ ), oxygen desaturations per hour ( $28.4 \pm 21.2$ versus $29.8 \pm 23$ versus $34.7 \pm 24.6$ ), mean arterial oxygen saturation $\left(S_{\mathrm{aO}_{2}}\right)$ dips $(88.3 \pm 7.5$ versus $88.1 \pm 4.5$ versus $87.6 \pm 5.3 \%)$, time spent with $S_{\mathrm{aO}_{2}}<90 \%$ ( $14.4 \pm 20.7$ versus $17.7 \pm 24$ versus $21.5 \pm 25.7 \%$ of the recording). After 1 year $65.7 \%$ ( $\left.\mathrm{n}=879\right)$ of our patients used CPAP at least $5 \mathrm{~h}$ per night, $11.6 \%(\mathrm{n}=155)$ between 4 and 4.9 and only $22 \%(\mathrm{n}=305)$ $<3.9 \mathrm{~h}$.per night. Adherence to treatment in the first week is not discriminant since patients do not receive a CPAP prescription until they show at least $4 \mathrm{~h}$ per night of usage.

Our study reports adherence to CPAP therapy after 1 year of treatment in consecutive patients in a clinical setting including an educational session. Our data suggest that a sustainable, patient centred programme can work in real life leading to adherence rates comparable or even better than more expensive and articulated interventions [3-8]. This is more important if we consider the cost of sleep disorders for the national health system.

Our study confirms data of Cistulli et al. [9], a study conducted on a far bigger sample (2.6 million versus 1300) of unselected patients using a unique device brand, relying on a more traditional definition of adherence and 90-day follow-up showing an adherence rate of about $75 \%$. Our study adopted a more restrictive adherence definition (at least $4 \mathrm{~h}$ every night), includes all patients who were prescribed CPAP in a clinical setting, used different CPAP brands and a standardised protocol. 1-year follow-up is a time-span offering more reliable information on long-term adherence. A limitation of both studies is that case series do not include those who refused treatment, who in our practice can rely on a tailored alternative therapeutic programme. The final message is that about four out of five patients who decide to start CPAP treatment have a satisfying adherence in the long term. We believe that a careful choice of interface and education of patients in the first week is crucial, but the opportunity to have quick access to the sleep laboratory when a problem occurs is another key to success: one out of five patients relied on this opportunity and new programmes of telemonitoring could help in assuring more timely interventions. Expensive tools and time-consuming programmes statistically increase the time on CPAP, but often this difference is below a clinically relevant threshold [8], or can lead to good results [10], but are difficult to apply on a larger scale. However, $22 \%$ of patients using CPAP for less than $4 \mathrm{~h}$ per night is still a critical rate and additional resources could be focused on these subjects more than in the effort to enhance adherence of patients already compliant to treatment. Future studies should focus on investigating the causes of the low adherence in this new scenario and construct a specific protocol on the basis of personality characteristics and co-morbidity, particularly when insomnia is the cause of an insufficient use.

In conclusion, our study suggests that nowadays adherence to CPAP therapy is effective when applying a standard protocol consisting of a diagnostic procedure, accurate pressure setting and mask choice, a group educational session on sleep and sleep disorders, and 1-year follow-up visit. Adherence rates are 
consistently high compared to the figures of a decade ago, possibly due to the improvement in technology and more opportunities for alternative treatments in less severe patients, supporting the hypothesis that a sustainable and accurate procedure for OSA patient management can work in real life.

Elisa Morrone ${ }^{1}$, Andrea Giordano ${ }^{2}$, Sonia Carli ${ }^{1}$, Dina Visca $\oplus^{3}$, Fabio Rossato ${ }^{1}$, Massimo Godio ${ }^{1}$, Elena Paracchini ${ }^{1}$, Sabrina Rossi ${ }^{1}$, Bruno Balbi ${ }^{1}$, Carlo Sacco ${ }^{1}$ and Alberto Braghiroli $\circledast^{1}$

${ }^{1}$ Sleep Laboratory, Dept Pulmonary Rehabilitation, ICS Maugeri Spa SB, Scientific Institute of Veruno, Veruno, Italy. ${ }^{2}$ Bioengineering Dept, ICS Maugeri Spa SB, Scientific Institute of Veruno, Veruno, Italy. ${ }^{3}$ Dept Pulmonary Rehabilitation, ICS Maugeri Spa SB, Scientific Institute of Tradate, Tradate, Italy.

Correspondence: Alberto Braghiroli, Istituti Clinici Scientifici Maugeri IRCCS, Via Maugeri 4, 27100 Pavia, Italy. E-mail: alberto.braghiroli@icsmaugeri.it

Received: 18 July 2019 | Accepted after revision: 10 Nov 2019

Support statement: The study was supported by the "Ricerca Corrente" funding of ICS Maugeri Spa SB. E. Morrone is recipient of a grant from ICS Maugeri Spa SB for scientific consulting.

Conflict of interest: None declared.

\section{References}

1 Young T, Palta M, Dempsey J, et al. The occurrence of sleep-disordered breathing among middle-aged adults. N Engl J Med 1993; 328: 1230-1235.

2 Bakker JP, Weaver TE, Parthasarathy S, et al. Adherence to CPAP what should we be aiming for, and how can we get there? Chest 2019; 155: 1272-1287.

3 Aloia MS, Smith K, Arnedt JT, et al. Brief behavioral therapies reduce early positive airway pressure discontinuation rates in sleep apnea syndrome: preliminary findings. Behav Sleep Med 2007; 5: 89-104.

4 Olsen S, Smith SS, Oei TP, et al. Motivational interviewing (MINT) improves continuous positive airway pressure (CPAP) acceptance and adherence: a randomized controlled trial. J Consult Clin Psychol 2012; 80: 151-163.

5 Aloia MS, Arnedt JT, Strand M, et al. Motivational enhancement to improve adherence to positive airway pressure in patients with obstructive sleep apnea: a randomized controlled trial. Sleep 2013; 36: 1655-1662.

6 Bakker JP, Wang R, Weng J, et al. Motivational enhancement for increasing adherence to CPAP: a randomized controlled trial. Chest 2016; 150: 337-345.

7 Hostler JM, Sheikh KL, Andrada TF, et al. A mobile, web-based system can improve positive airway pressure adherence. J Sleep Res 2017; 26: 139-146.

8 Pépin JL, Jullian-Desayes I, Sapene M, et al. Multimodal remote monitoring of high cardiovascular risk OSA patients initiating CPAP: a randomized trial. Chest 2019; 155: 730-739.

9 Cistulli PA, Armitstead J, Pépin JL, et al. Short-term CPAP adherence in obstructive sleep apnea: a big data analysis using real world data. Sleep Med 2019; 59: 114-116.

10 Pépin JL, Krieger J, Rodenstein D, et al. Effective compliance during the first 3 months of continuous positive airway pressure. A European prospective study of 121 patients. Am J Respir Crit Care Med 1999; 160: 1124-1129. 